\title{
Efeito da impregnação a vácuo na transferência de massa durante o processo de salga de cortes de peito de frango
}

\author{
Effect of vacuum impregnation on mass transfer during the salting process of chicken breast cuts
}

Franciny Campos SCHMIDT ${ }^{1}$, Bruno Augusto Mattar CARCIOFI ${ }^{1}$, João Borges LAURINDO ${ }^{1 \star}$

\section{Resumo}

A impregnação a vácuo (IV) tem sido estudada como uma alternativa para reduzir o tempo dos processos de salga aplicados a diversos alimentos. Neste trabalho, foi investigada a influência da aplicação de vácuo no processo de salga de cortes de peito de frango. Os cortes foram submersos em soluções com diferentes concentrações de $\mathrm{NaCl}$ para a avaliação de dois processos distintos de impregnação de sal: a) processo inteiramente a pressão atmosférica (IPA); e b) com aplicação de vácuo seguido do restabelecimento da pressão atmosférica (IV). A transferência de massa entre a amostra e a solução salina foi avaliada através das determinações de ganho de água (GA), ganho de sal (GS) e ganho de massa total (GM) pelas amostras submetidas à IV e à IPA. A comparação entre os processos de IV e IPA, com 6 horas de imersão, indicou que a utilização de um período inicial de vácuo pode incrementar o GA, GS e GM em 78, 25 e 54\%, respectivamente. Isso se deve à contribuição sinérgica do mecanismo hidrodinâmico (HDM) aos mecanismos osmóticos e difusivos existentes. Deste modo, a IV pode ser considerada como uma alternativa de processo para a salga de cortes de carne de frango. No entanto, deve-se estar atento para que os ganhos de água e sal sejam compatíveis com as exigências legais e tecnológicas.

Palavras-chave: transferência de massa; impregnação a vácuo; processo de salga.

\begin{abstract}
Vacuum impregnation has been studied as an alternative for reducing time in the salting process applied to different kinds of food. In this study, the influence of vacuum application on the salting process of chicken breast cuts was evaluated. The chicken samples were submerged in solutions with different $\mathrm{NaCl}$ concentrations and two processes were evaluated: a) a process entirely under atmospheric pressure (API); and $b$ ) a process with vacuum application followed by atmospheric pressure restoration (VI). Mass transfers were characterized by water gain (WG), salt gain (SG), and total weight increment (WI). The comparison between the VI and API processes, with 6 hours of immersion time, indicated that the utilization of an initial vacuum period can increase the WG, SG and WI in 78, 25 and $54 \%$, respectively. This is due to the synergistic contribution of the hydrodynamic mechanisms (HDM) to the existing osmotic and diffusive mechanisms. Thus, VI can be considered as an alternative for the salting process of chicken cuts. However, special care must be taken in order to assure that water and salt increments are compatible with legal and technological requirements.

Keywords: mass transfer; vacuum impregnation; salting process.
\end{abstract}

\section{Introdução}

O processo de impregnação a vácuo (IV) de alimentos porosos consiste na troca do gás e do líquido nativos presentes no interior dos poros por um líquido externo. Isso se faz pela ação de gradientes macroscópicos de pressão promovidos pela aplicação de vácuo, seguida pelo restabelecimento da pressão atmosférica em uma câmara contendo o produto imerso em uma solução (FITO, 1994; FITO et al., 1996; FITO; PASTOR, 1994). No primeiro passo do processo de IV, submete-se o sistema a uma pressão subatmosférica $\left(\mathrm{P}_{1}\right)$ por um dado período de tempo $\left(\mathrm{t}_{1}\right)$, em que o gás ocluso nos poros se expande até o equilíbrio com a pressão imposta ao sistema, saindo do produto e drenando parte do líquido nativo presente no interior dos poros. Uma vez alcançado o equilíbrio das pressões do sistema, uma quantidade de líquido penetra nos poros devido à ação das forças capilares. Após este período, a pressão atmosférica $\left(\mathrm{P}_{2}\right)$ é restabelecida e o produto é mantido imerso na solução por um tempo $t_{2}$. Nesta segunda etapa, o gás residual presente no interior da amostra é comprimido, conduzindo a impregnação dos poros do produto pela solução externa (FITO, 1994; FITO et al., 1996; 2001). Esse fenômeno implica uma mudança composicional rápida no produto, com modificação das condições para a transferência de massa (FITO et al., 1996). Fito (1994) propôs o primeiro modelo matemático destinado à predição da impregnação a vácuo, denominado pelo autor de Mecanismo Hidrodinâmico (HDM).

A utilização de vácuo tem sido estudada para reduzir o tempo dos processos de salga aplicados a queijos (ANDRÉS, 1995; FITO; PASTOR, 1994), presunto (BARAT et al., 2005)

Recebido para publicação em 22/1/2007

Aceito para publicação em 15/10/2007 (002221)

Departamento de Engenharia Química e Engenharia de Alimentos, Universidade Federal de Santa Catarina - UFSC, CP 476, CEP 88040-900, Florianópolis - SC, Brasil,

E-mail: joao@enq.ufsc.br

${ }^{*}$ A quem a correspondência deve ser enviada 
e peixes e carnes (CHIRALT et al., 2001; DEUMIER et al., 2003a; b; SCHMIDT, 2006). Os resultados relatados nesses trabalhos mostraram que o processo de IV promoveu uma melhor distribuição de sal no produto quando comparado ao processo de impregnação a pressão atmosférica. O ganho de sal é acelerado pela combinação do HDM com os fenômenos difusivos promovidos pelos gradientes de concentração entre o produto e a solução (CHIRALT et al., 2001). Neste trabalho estudou-se a influência da aplicação de vácuo no ganho de água (GA), ganho de sal (GS) e ganho de massa total (GM) por cortes de peito de frango durante o processo de salga por imersão.

\section{Material e métodos}

\subsection{Carne de frango e soluções salinas}

Cortes de peito de frango resfriados, sem osso e sem pele, provenientes da mesma unidade produtora e adquiridos no comércio local de Florianópolis - SC foram utilizados para preparação das amostras. As mesmas foram cortadas em formato de paralelepípedo de 4,0 ×3,0 × 1,5 cm, com peso variando entre $18 \mathrm{e} 20 \mathrm{~g}$. A umidade inicial foi determinada pelo método gravimétrico (secagem em estufa a $105^{\circ} \mathrm{C}$ por 24 horas). As amostras foram condicionadas à temperatura dos experimentos $\left(5^{\circ} \mathrm{C}\right)$ até sua utilização. As soluções salinas foram preparadas com cloreto de sódio ( $\mathrm{NaCl}$, P.A.) e água destilada. Uma razão mássica de 1:50 de frango: salmoura foi utilizada para evitar mudanças significativas na concentração de sal durante os ensaios.

\subsection{Dispositivo experimental}

O dispositivo experimental, esquematizado na Figura 1, consiste em uma câmara encamisada com controle e monitoramento de temperatura. O controle de temperatura foi realizado através da circulação de água proveniente de um banho termostatizado (MICROQUÍMICA, Modelo MQBMP-01, Florianópolis, Brasil). A câmara foi conectada a uma bomba de vácuo (VEB, Modelo A DS 30, Alemanha) e a um transmissor de pressão (IOPE, Modelo TWPI, Série 8388, Brasil). Este último foi conectado a um microcomputador, possibilitando o monitoramento on-line da pressão da câmara através de um programa de aquisição de dados dedicado a esse fim. No interior da câmara as amostras foram acondicionadas entre duas telas de metal, que impediam que as mesmas flutuassem durante os experimentos. A agitação da solução foi realizada através de duas bombas submersas de circulação de líquidos (ATMAN, Modelo AT 005, USA).

\subsection{Metodologia}

No primeiro estudo, as amostras foram submersas nas soluções salinas com diferentes concentrações de sal: 5, 10, 15 e $20 \%$ (g de sal. $100 \mathrm{~g}^{-1}$ de salmoura). Foram comparados dois processos: a) inteiramente a pressão atmosférica (IPA) por 15 minutos; e b) com aplicação de vácuo (IV) por 15 minutos, com pressão absoluta de $10 \mathrm{mmHg}\left(\mathrm{P}_{1}\right)$, seguida do restabelecimento instantâneo da pressão atmosférica $\left(\mathrm{P}_{2}\right)$ e retirada imediata das amostras.

No segundo estudo foi avaliada a cinética de transferência de massa para os processos de IPA e IV. As amostras de peito de frango foram submersas em solução salina com $10 \%$ em massa de $\mathrm{NaCl}$. Foram avaliados os processos: a) inteiramente a pressão atmosférica (IPA) por 24 horas; e b) com aplicação de vácuo $\left(\mathrm{P}_{1}=10 \mathrm{mmHg}\right)$ por 15 minutos seguida de 5,75 horas a pressão atmosférica (IV).

Todos os experimentos foram realizados a $5^{\circ} \mathrm{C}$. A transferência de massa (lato sensu) foi caracterizada pela determinação dos parâmetros relativos ao ganho de água (GA), ganho de sal (GS) e ganho de massa total (GM).

\section{Determinação do GA, GS e GM}

Para tempos de imersão pré-determinados, as amostras foram retiradas e a salmoura aderida na superfície foi removida com o uso de papel filtro. Cada amostra foi pesada, triturada e homogeneizada. Tomaram-se alíquotas para determinação dos teores de umidade (método gravimétrico, $105^{\circ} \mathrm{C}, 24$ horas) e das concentrações de $\mathrm{NaCl}$, através da quantificação de cloretos, de acordo com o Método de Möhr (BRASIL, 1999). Todas as determinações foram realizadas em triplicata. Os parâmetros ganho de água (GA), ganho de sal (GS) e ganho de massa total

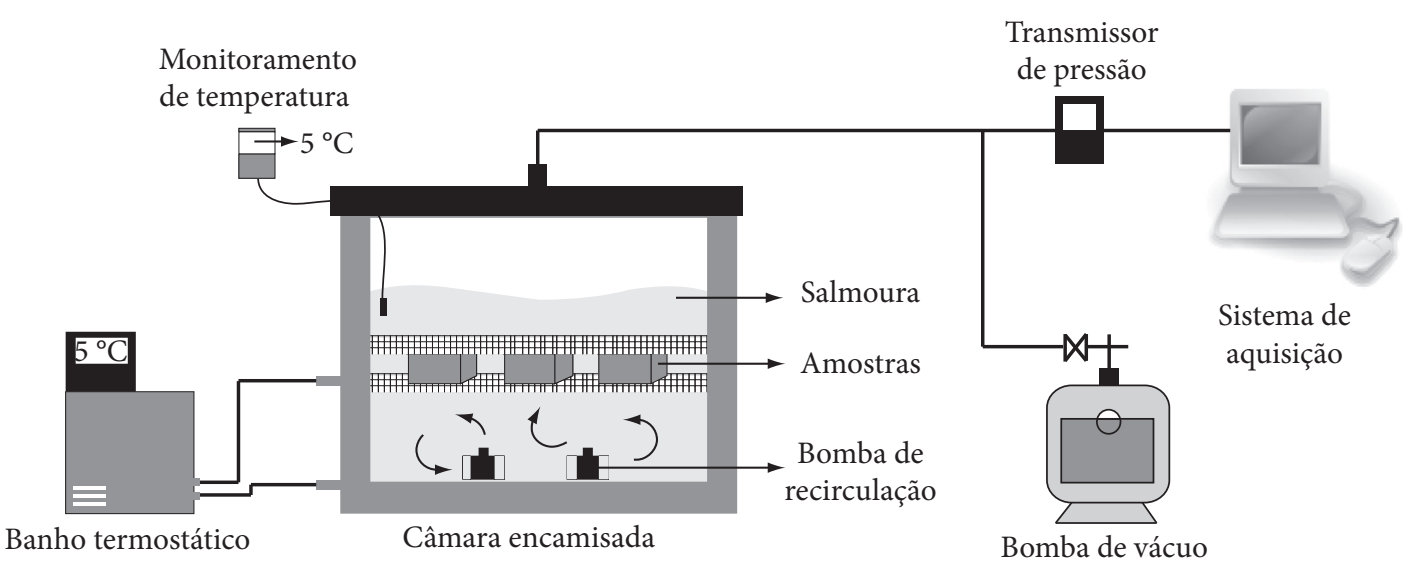

Figura 1. Representação esquemática do dispositivo experimental utilizado. 
(GM) foram calculados através das Equações 1, 2 e 3, respectivamente, e expressos em gramas por $100 \mathrm{~g}$ de amostra inicial (g.100 g-1).

$$
\begin{aligned}
& \mathrm{GA}=\frac{m_{a}-m_{a o}}{m_{o}} \times 100 \\
& \mathrm{GS}=\frac{m_{s}-m_{s}}{m_{o}} \times 100 \\
& \mathrm{GM}=\frac{m-m_{o}}{m_{o}} \times 100
\end{aligned}
$$

em que: $m_{\mathrm{a}}$ é a massa de água da amostra no tempo t; $m_{\text {ao }}$ é a massa inicial de água da amostra; $m_{\mathrm{o}}$ é a massa inicial da amostra; $m$ é a massa da amostra no tempo t; $m_{\mathrm{s}}$ é a massa de sal no tempo t; e $m_{\text {so }}$ é a massa inicial de sal na amostra.

\subsection{Modelagem matemática}

O modelo empírico proposto por Azuara et al. (1992) foi utilizado para representar a cinética de transferência de massa durante os processos de salga realizados a pressão atmosférica (IPA) e com aplicação de vácuo (IV). O modelo foi desenvolvido para representar a cinética do processo osmótico e estimar a concentração de equilíbrio a partir do balanço de massa no produto. O balanço de massa (água, sal ou massa total) no produto pode ser expresso como:

$G X=G X^{\infty}-G X^{*}$

em que:

GX é o ganho de água (GA), de sal (GS) ou de massa total (GM) pelo produto ocorrido até o tempo t; $G X^{\infty}$ é o ganho de água, de sal ou de massa total no equilíbrio; e $G X^{\star}$ é a massa de sal ou de água que ainda não entrou (ou saiu) da amostra no tempo $t$, mas que irá entrar até que o equilíbrio seja alcançado.

O ganho de massa no equilíbrio $\left(G X^{\infty}\right)$ depende da temperatura e da concentração da salmoura, enquanto $G X$ e $G X^{\star}$ dependem do tempo de processo. O valor do $G X$ aumenta e o valor do $G X^{\star}$ diminui durante o processo osmótico, permitindo estabelecer uma relação entre essas variáveis, representada por um parâmetro K. Por sua vez, esse parâmetro é função do tempo e da taxa de ganho de massa (água, sal ou massa total), podendo ser escrito em termos do tempo de processo t, e de uma constante k, conforme representado pela Equação 5 (AZUARA et al., 1992).

$$
G X^{*}=\frac{G X}{\mathrm{~K}}=\frac{G X}{\mathrm{k} \cdot \mathrm{t}}
$$

Substituindo a Equação 5 na Equação 4 e rearranjando os termos têm-se:

$$
G X=\frac{\mathrm{k} \cdot \mathrm{t}\left(G X^{\infty}\right)}{1+\mathrm{k} \cdot \mathrm{t}}
$$

A Equação 6 pode ser utilizada para caracterizar o processo osmótico de diferentes tipos de alimentos, sem restrições da configuração geométrica (AZUARA et al., 1992). O ajuste da Equação 6 aos dados experimentais do GA, GS e GM, para ambos os processos em estudo (IPA e IV), foi realizado com o auxílio do software MATLAB $^{\circledR}$ R2006a.

Os valores do ganho de massa (água, sal ou massa total) no equilíbrio $\left(G X^{\infty}\right)$ e do parâmetro $k$ foram calculados através da regressão linear do modelo proposto por Azuara et al. (1992), utilizando os dados experimentais dos ganhos de massa obtidos durante o processo osmótico. A forma linear para a Equação 6 é dada por:

$$
\frac{\mathrm{t}}{G X}=\frac{1}{k\left(G X^{\infty}\right)}+\frac{\mathrm{t}}{G X^{\infty}}
$$

\subsection{Análise estatística}

As diferenças entre os valores do ganho de água (GA), ganho de sal (GS) e ganho de massa total (GM), obtidas no processo inteiramente a pressão atmosférica (IPA) e com aplicação de vácuo (IV), foram avaliadas através da Análise de Variância (ANOVA) com nível de significância de $5 \%(\alpha=0,05)$, com o auxílio do software STATISTICA 6.0.

A adequação do modelo proposto por Azuara et al. (1992) aos dados experimentais foi avaliada pelo coeficiente de correlação $\left(\mathrm{R}^{2}\right)$ e pelo erro médio quadrático (MSE), conforme a Equação 8.

$$
\mathrm{MSE}=\frac{\sum_{n}\left(G X_{\text {predito }}-G X_{\text {exp }}\right)^{2}}{n}
$$

em que: $G X_{\text {predito }}$ e $G X_{\text {exp }}$ são, respectivamente, os valores do ganho de massa (água, sal ou massa total) predito e experimental, e $n$ o número de medidas experimentais.

\section{Resultados e discussão}

As comparações entre os valores do ganho de massa total (GM) obtidos para o processo inteiramente a pressão atmosférica (IPA) e para o processo de impregnação a vácuo (IV), realizadas com diferentes concentrações de salmoura $\left(C_{s}\right)$, são apresentadas na Figura 2. Os dois processos tiveram duração total de 15 minutos e a pressão aplicada durante a IV foi de $10 \mathrm{mmHg}$. Os valores do ganho de massa total nos processos de IV e IPA foram significativamente diferentes $(\mathrm{p}<0,05)$ para todas as $\mathrm{C}_{\mathrm{s}}$ analisadas. Essas diferenças são devido à ação do mecanismo hidrodinâmico, resultante dos gradientes macroscópicos de pressão. Esses gradientes ocorrem devido à aplicação de vácuo e postezrior restabelecimento da pressão atmosférica, o que leva ao preenchimento dos espaços intercelulares do tecido muscular pela salmoura.

Para o processo de salga inteiramente a pressão atmosférica com $\mathrm{C}_{\text {s }}$ de $20 \%$ de $\mathrm{NaCl}$, observou-se perda de massa pelas amostras $(\mathrm{GM}<0)$. Isso foi resultado da perda de água através do mecanismo osmótico e da solubilização de proteínas miofibrilares, que prevaleceram em relação aos ganhos de sal por difusão e de solução salina (água e sal) por capilaridade. No entanto, ao final do processo de IV com $\mathrm{C}_{\mathrm{s}}$ de $20 \%$ de $\mathrm{NaCl}$, foi observado um ganho de massa pelas amostras $(G M>0)$. Neste caso, os poros da carne (espaços intercelulares) são preenchidos pela salmoura devido à ação do HDM, superando as perdas de 


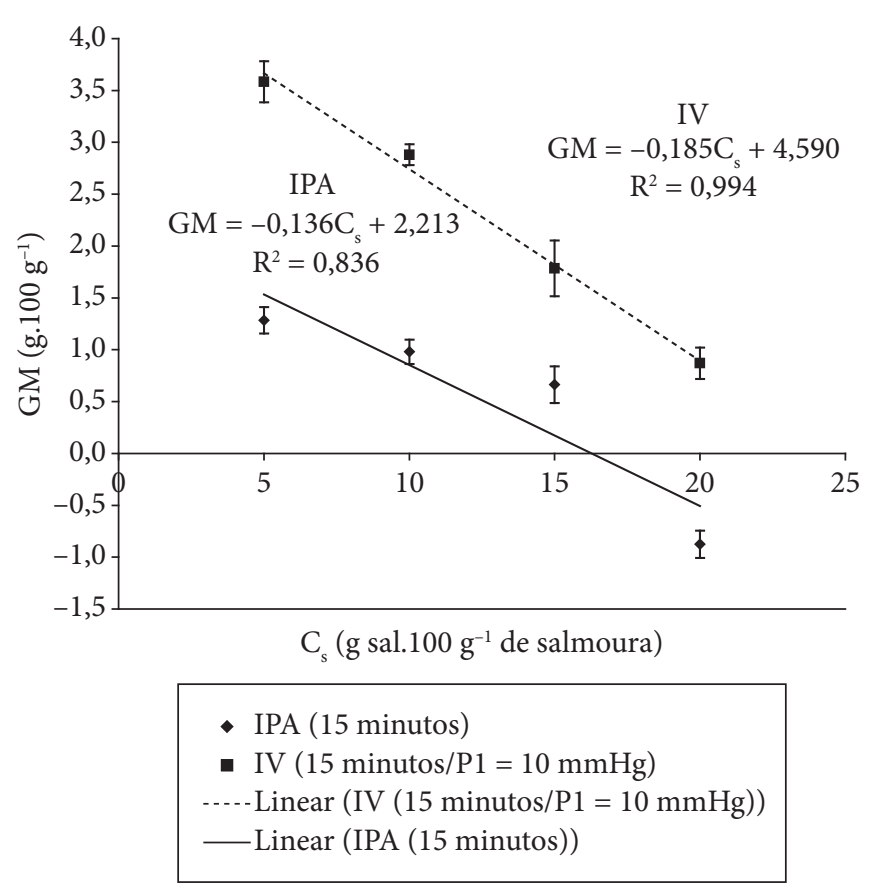

Figura 2. Comparação do ganho de massa total (GM) para IV e IPA utilizando diferentes concentrações de salmoura no processo de salga de cortes de peito de frango a $5{ }^{\circ} \mathrm{C}$. ( $\left.\diamond\right) \mathrm{IPA}(\mathrm{P}=760 \mathrm{mmHg} / 15$ minutos); (घ) IV ( $\mathrm{P}=10 \mathrm{mmHg} / 15$ minutos).

água (desidratação osmótica) e de proteínas solúveis. Para as demais $\mathrm{C}_{\mathrm{s}}$, os incrementos dos valores de GM para IV foram sempre superiores aos valores obtidos com o processo de IPA. Especificamente, os valores de GM obtidos para $\mathrm{C}_{\mathrm{s}}$ de 5, $10 \mathrm{e}$ $15 \%$ de $\mathrm{NaCl}$ foram cerca de 2,8 vezes maiores para o processo de IV. Ainda na Figura 2, pode-se observar que apenas para o processo de IV foi possível assumir um comportamento linear do GM em função da $C_{\mathrm{s}}$ com um bom ajuste $\left(\mathrm{R}^{2}=0,994\right)$.

Na Figura 3 é apresentada a comparação dos valores do ganho de água (GA) por amostras tratadas por IPA e IV. Observase que a IV aumentou significativamente $(\mathrm{p}<0,05)$ o GA pelas amostras imersas em solução com 5 e $10 \%$ de NaCl. Mesmo para $\mathrm{C}_{\mathrm{s}}$ de $15 \% \mathrm{de} \mathrm{NaCl}$, as amostras submetidas à IV apresentaram um $\mathrm{GA}>0$, superando a perda de água por osmose, observada no processo de IPA. Para $\mathrm{C}_{\mathrm{s}}$ de $20 \%$, a perda de água foi significativamente menor para a IV $(\mathrm{p}<0,05)$, devido à compensação de parte das perdas por osmose pelo mecanismo hidrodinâmico (HDM). De modo geral, as maiores quantidades de água nas amostras tratadas pela IV são resultado do preenchimento dos poros pela solução devido aos gradientes de pressão.

Através do ajuste dos valores do ganho de água (GA) em função da concentração da salmoura $\left(\mathrm{C}_{\mathrm{s}}\right)$, observou-se um comportamento linear para os processos a pressão atmosférica (IPA) e a vácuo (IV), com coeficientes de correlação $\left(\mathrm{R}^{2}\right)$ de 0,991 e 0,996, respectivamente (Figura 3). A partir do ajuste linear do GA em função da $\mathrm{C}_{\mathrm{s}}$ apresentado na Figura 3, foi possível estimar a transição do regime de hidratação e desidratação osmótica para o processo de IPA e IV com tempo de imersão de 15 minutos. A concentração de salmoura $\left(\mathrm{C}_{\mathrm{s}}\right)$ referente à

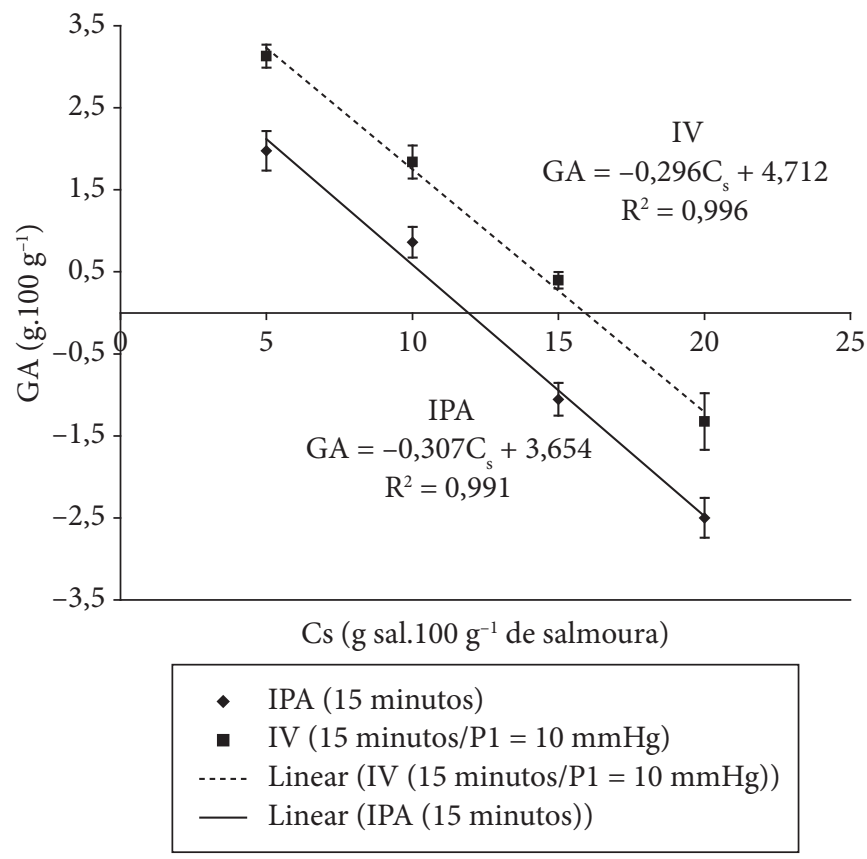

Figura 3. Comparação do ganho de água (GA) para IV e IPA utilizando diferentes concentrações de salmoura no processo de salga de cortes de peito de frango a $5{ }^{\circ} \mathrm{C}$. ( $\left.\diamond\right)$ IPA ( $\mathrm{P}=760 \mathrm{mmHg} / 15$ minutos); ( $\left.\mathbf{\square}\right)$ $\mathrm{IV}(\mathrm{P}=10 \mathrm{mmHg} / 15$ minutos $)$.

transição entre o regime de hidratação e o regime de desidratação foi de aproximadamente $12 \mathrm{~g}$ sal. $100 \mathrm{~g}^{-1}$ de salmoura para o processo a pressão atmosférica e de $16 \mathrm{~g}$ sal. $100 \mathrm{~g}^{-1}$ de salmoura para o processo de impregnação a vácuo.

Na Figura 4 são apresentados os dados do ganho de sal (GS) obtidos nos processos de salga inteiramente a pressão atmosférica (IPA) e com aplicação de vácuo (IV). Através da análise de variância (ANOVA) do GS entre os processos de IPA e IV, foram observadas diferenças significativas apenas para os tratamentos com $\mathrm{C}_{\mathrm{s}}$ de 15 e $20 \%$. Para estes tratamentos, o ganho de sal obtido no processo de IV foi em média $27 \pm 1 \%$ maior que o ganho de sal no processo de IPA. Para os tratamentos com $\mathrm{C}_{\mathrm{s}}$ de 5 e $10 \%$, não foi observada diferença significativa $(p>0,05)$ no GS entre os processos. Provavelmente, para soluções salinas mais diluídas, as diferenças estatísticas estão mais próximas dos erros nas determinações experimentais.

Ainda na Figura 4, observa-se o comportamento linear do GS em função da $\mathrm{C}_{\mathrm{s}}$ para ambos os processos em estudo, com coeficientes de correlação $\left(R^{2}\right)$ de 0,978 para o processo a pressão atmosférica e de 0,986 para o processo de impregnação a vácuo. Este comportamento linear também foi observado por Deumier et al. (2003b) em experimentos de salga de cortes de peito de peru a pressão atmosférica.

Assim, através dos ajustes lineares apresentados na Figura 4, pode-se estimar o ganho de sal pelos cortes de peito de frango imersos em soluções salinas com concentração na faixa de 5 a $0 \%$ de $\mathrm{NaCl}$, nas condições estudadas. 


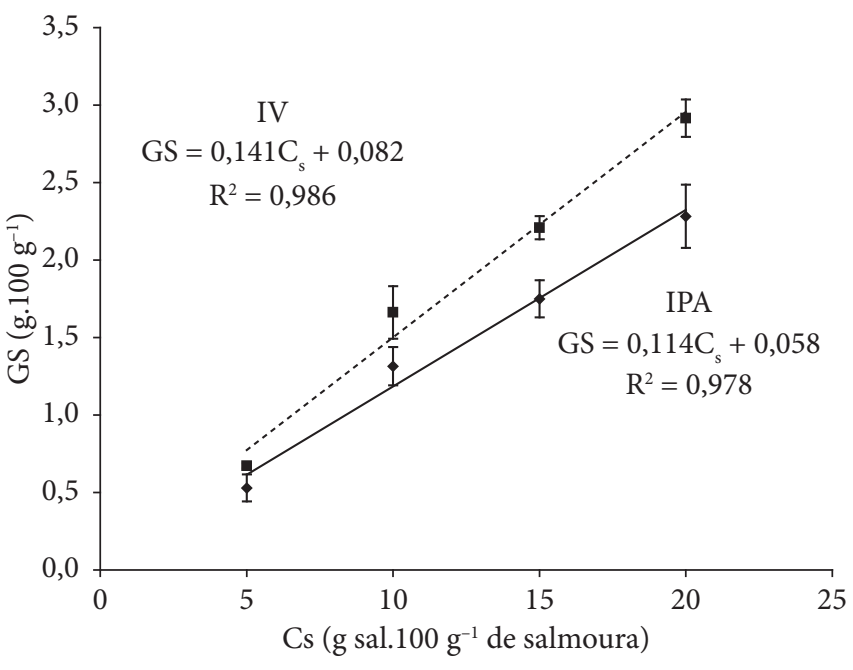

- IPA (15 minutos)

- IV (15 minutos/P1 = $10 \mathrm{mmHg})$

-.-.- Linear (IV (15 minutos/P1 = $10 \mathrm{mmHg})$ )

— Linear (IPA (15 minutos))

Figura 4. Comparação do ganho de sal (GS) para IV e IPA utilizando diferentes concentrações de salmoura no processo de salga de cortes de peito de frango a $5{ }^{\circ} \mathrm{C}$. ( $\bullet$ IPA $(\mathrm{P}=760 \mathrm{mmHg} / 15$ minutos); ( IV $(\mathrm{P}=10 \mathrm{mmHg} / 15$ minutos $)$.

Nas Figuras 5a, 5b e 5c são apresentadas as cinéticas de ganho de água (GA), ganho de sal (GS) e ganho da massa total (GM), para os cortes de peito de frango submetidos ao processo de salga inteiramente a pressão atmosférica - IPA ( $\mathrm{P}=760 \mathrm{mmHg}$, durante 24 horas) e ao processo com aplicação de vácuo - IV ( $\mathrm{P}_{1}=10 \mathrm{mmHg}$ para os primeiros 15 minutos e $\mathrm{P}_{2}=760 \mathrm{mmHg}$ para as 5,75 horas subseqüentes). Na avaliação da cinética, a concentração de salmoura utilizada em ambos os processos foi de $10 \%$ de $\mathrm{NaCl}$ (g sal.100 $\mathrm{g}^{-1}$ de salmoura).

Ainda nas Figuras 5a, 5b e 5c são apresentados os ajustes do modelo proposto por Azuara et al. (1992) aos dados experimentais. A Tabela 1 apresenta os valores dos ganhos de massa (água, sal e massa total) no equilíbrio e os valores do parâmetro $\mathrm{k}$, determinados pela Equação 7, além dos parâmetros estatísticos relativos ao ajuste do modelo ( $\mathrm{R}^{2}$ e $\mathrm{MSE}$ ). Observa-se que o modelo apresentou um bom ajuste aos dados experimentais do processo a pressão atmosférica ( $\mathrm{R}^{2}$ entre 0,946 e 0,967$)$. No entanto, para o processo de IV o ajuste foi um pouco inferior ( $\mathrm{R}^{2}$ entre 0,822 e 0,942 ), em virtude da menor quantidade de pontos experimentais para longos tempos de processo.

$\mathrm{Na}$ Figura 5a pode-se observar diferença significativa $(\mathrm{p}<0,05)$ no ganho de água $(\mathrm{GA})$ das amostras submetidas à IV em relação ao ganho obtido através da IPA, durante todo o intervalo de tempo analisado. Em 6 horas de imersão, as amostras submetidas ao processo com aplicação de vácuo (IV) apresentaram um GA de 6,6 g água.100 $\mathrm{g}^{-1}$ de amostra inicial, enquanto que a pressão atmosférica (IPA) o GA foi de $3,7 \mathrm{~g}$ água. $100 \mathrm{~g}^{-1}$ de amostra inicial.
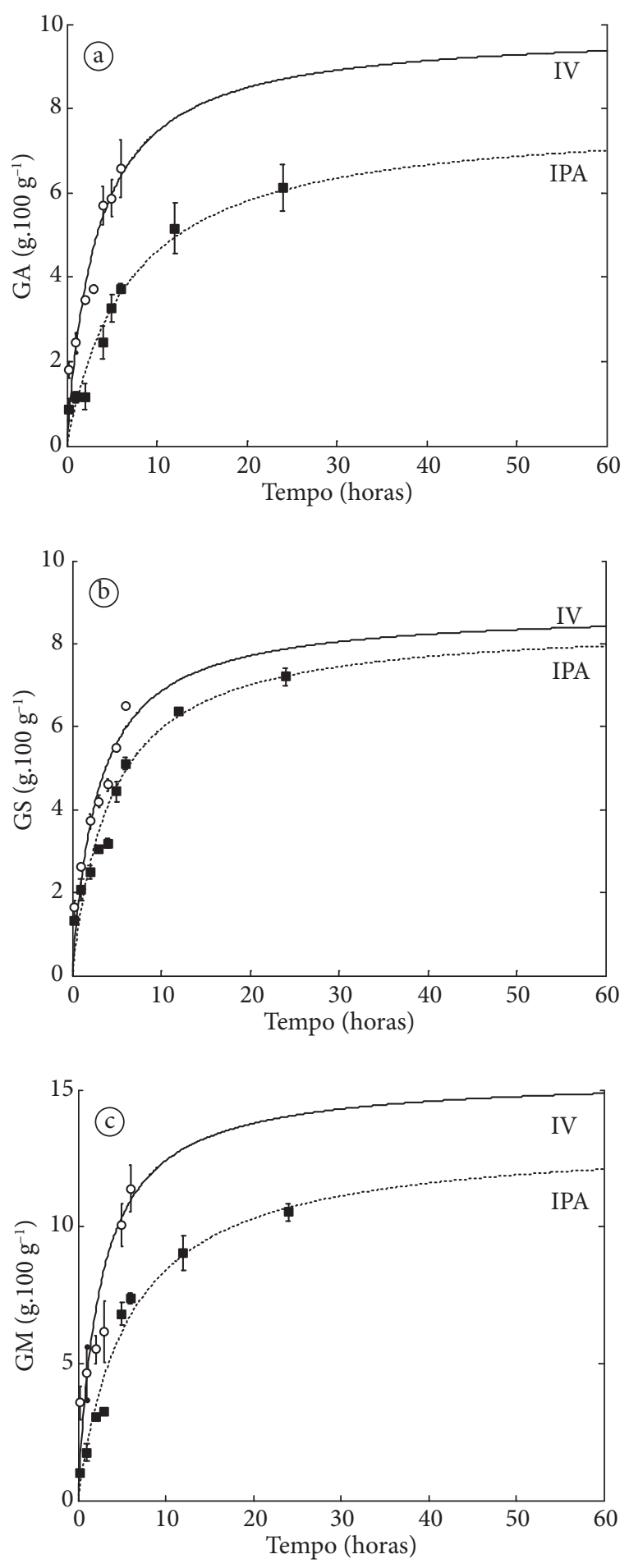

- Dados experimentais IPA ... Modelo empírico, Equação 6 IPA

- Dados experimentais IV

— Modelo empírico, Equação 6 IV

Figura 5. Efeito da aplicação de vácuo em: a) ganho de água (GA); b) ganho de sal (GS); e c) ganho de massa total (GM) pelos cortes de peito de frango imersos em solução com $10 \%$ de $\mathrm{NaCl}$ a $5{ }^{\circ} \mathrm{C}$ e ajuste do modelo proposto por Azuara et al. (1992). ( $\mathrm{O}) \mathrm{IPA}(\mathrm{P}=760 \mathrm{mmHg} ; \mathrm{t}=6$ horas $)(\mathbf{\square})$ IV ( $\mathrm{P}_{1}=10 \mathrm{mmHg} ; \mathrm{t}_{1}=15$ minutos; $\mathrm{P}_{2}=760 \mathrm{mmHg} ; \mathrm{t}_{2}=5,75$ horas $)$. 
Para o ganho de sal (GS), apresentado na Figura 5b, só foi possível observar diferença significativa $(p<0,05)$ entre os dois processos (IPA e IV) a partir da $4^{a}$ hora de imersão. Em 6 horas de imersão, o valor do GS para o processo de IPA foi de $5,2 \mathrm{~g}$ sal. $100 \mathrm{~g}^{-1}$ de amostra inicial, enquanto que para a IV esse valor foi de $6,5 \mathrm{~g}$ sal. $100 \mathrm{~g}^{-1}$ de amostra inicial, indicando um aumento significativo $(\mathrm{p}<0,05)$ de $25 \%$ no ganho de sal. Este aumento no GS pelas amostras devido à aplicação de vácuo também foi observado por Deumier et al. (2003a) no processo de salga de cortes de peito de peru, por Barat et al. (2003) na salga de bacalhau e por Pezantes (2006) no processo de desidratação osmótica de filés de peixe. Na impregnação a vácuo, o preenchimento dos poros com a solução externa promove o aumento da área de contato entre a salmoura e o tecido muscular (interior da amostra), como esquematizado na Figura 6, o que sugere que a área disponível para transferência de massa no processo de IV é muito maior que no processo de IPA. No processo de salga inteiramente a pressão atmosférica (IPA), a área disponível para transferência é basicamente a superfície do corte de carne, enquanto no processo de impregnação a vácuo (IV) essa área é aumentada com a impregnação dos poros. Assim, o mecanismo difusivo atua no sentindo de homogeneizar as concentrações de sal na amostra, através de caminhos difusivos mais curtos.

$\mathrm{Na}$ Figura $5 \mathrm{c}$ pode-se observar a influência do mecanismo hidrodinâmico (HDM) na transferência de massa total (água + sal + proteínas solúveis) entre a solução salina e o produto. Em 6 horas de imersão, o valor do ganho de massa total (GM) para o processo de impregnação a vácuo foi de 11,4 g. $100 \mathrm{~g}^{-1} \mathrm{de}$ amostra inicial, enquanto que a pressão atmosférica o GM foi de 7,4 g. $100 \mathrm{~g}^{-1}$ de amostra inicial. Conforme o Regulamento Técnico de Qualidade e Identidade de Aves Temperadas do Ministério da Agricultura, o limite de incorporação de salmoura à carne de frango temperada é de $20 \%$. Deste modo, os valores de ganho de massa total obtidos nos processos de IPA e IV, com 6 horas de imersão, estão de acordo com limite estipulado pela legislação (BRASIL, 1999).

Tabela 1. Valores dos parâmetros estimados pelo modelo proposto por Azuara et al. (1992) e parâmetros estatísticos do ajuste do modelo aos dados experimentais do GA, GS e GM para os processos de IPA e IV.

\begin{tabular}{|c|c|c|c|c|c|c|c|c|c|c|c|c|}
\hline & \multicolumn{4}{|c|}{ Ganho de água } & \multicolumn{4}{|c|}{ Ganho de sal } & \multicolumn{4}{|c|}{ Ganho de massa total } \\
\hline & \multicolumn{2}{|c|}{$\begin{array}{c}\text { Parâmetros do } \\
\text { modelo }^{\mathrm{a}}\end{array}$} & \multicolumn{2}{|c|}{$\begin{array}{l}\text { Parâmetros } \\
\text { estatísticos }^{\mathrm{b}}\end{array}$} & \multicolumn{2}{|c|}{$\begin{array}{c}\text { Parâmetros do } \\
\text { modelo }^{\mathrm{a}}\end{array}$} & \multicolumn{2}{|c|}{$\begin{array}{l}\text { Parâmetros } \\
\text { estatísticos }\end{array}$} & \multicolumn{2}{|c|}{$\begin{array}{c}\text { Parâmetros do } \\
\text { modelo }^{\mathrm{a}}\end{array}$} & \multicolumn{2}{|c|}{$\begin{array}{l}\text { Parâmetros } \\
\text { estatísticos }^{\mathrm{b}}\end{array}$} \\
\hline & $\mathrm{GA}^{\infty}$ & $\mathrm{k}$ & $\mathrm{R}^{2}$ & MSE & $\mathrm{GS}^{\infty}$ & $\mathrm{k}$ & $\mathrm{R}^{2}$ & MSE & $\mathrm{GM}^{\infty}$ & $\mathrm{k}$ & $\mathrm{R}^{2}$ & MSE \\
\hline IPA & 7,825 & 0,143 & 0,966 & 0,115 & 8,525 & 0,230 & 0,946 & 0,229 & 13,263 & 0,173 & 0,967 & 0,366 \\
\hline IV & 9,881 & 0,305 & 0,909 & 0,353 & 8,818 & 0,348 & 0,942 & 0,235 & 15,480 & 0,403 & 0,822 & 2,039 \\
\hline
\end{tabular}

a Parâmetros estimados pelo modelo de Azuara et al. (1992): ponto no equilíbrio (GA ${ }^{\infty}, \mathrm{GS}^{\infty}$ e $\mathrm{GM}^{\infty}$ em g.100 g ${ }^{-1}$ ) e constante k; e ${ }^{\mathrm{b}}$ parâmetros estatísticos do ajuste do modelo de Azuara et al. (1992) aos dados experimentais: coeficiente de correlação $\left(R^{2}\right)$ e erro médio quadrático (MSE).
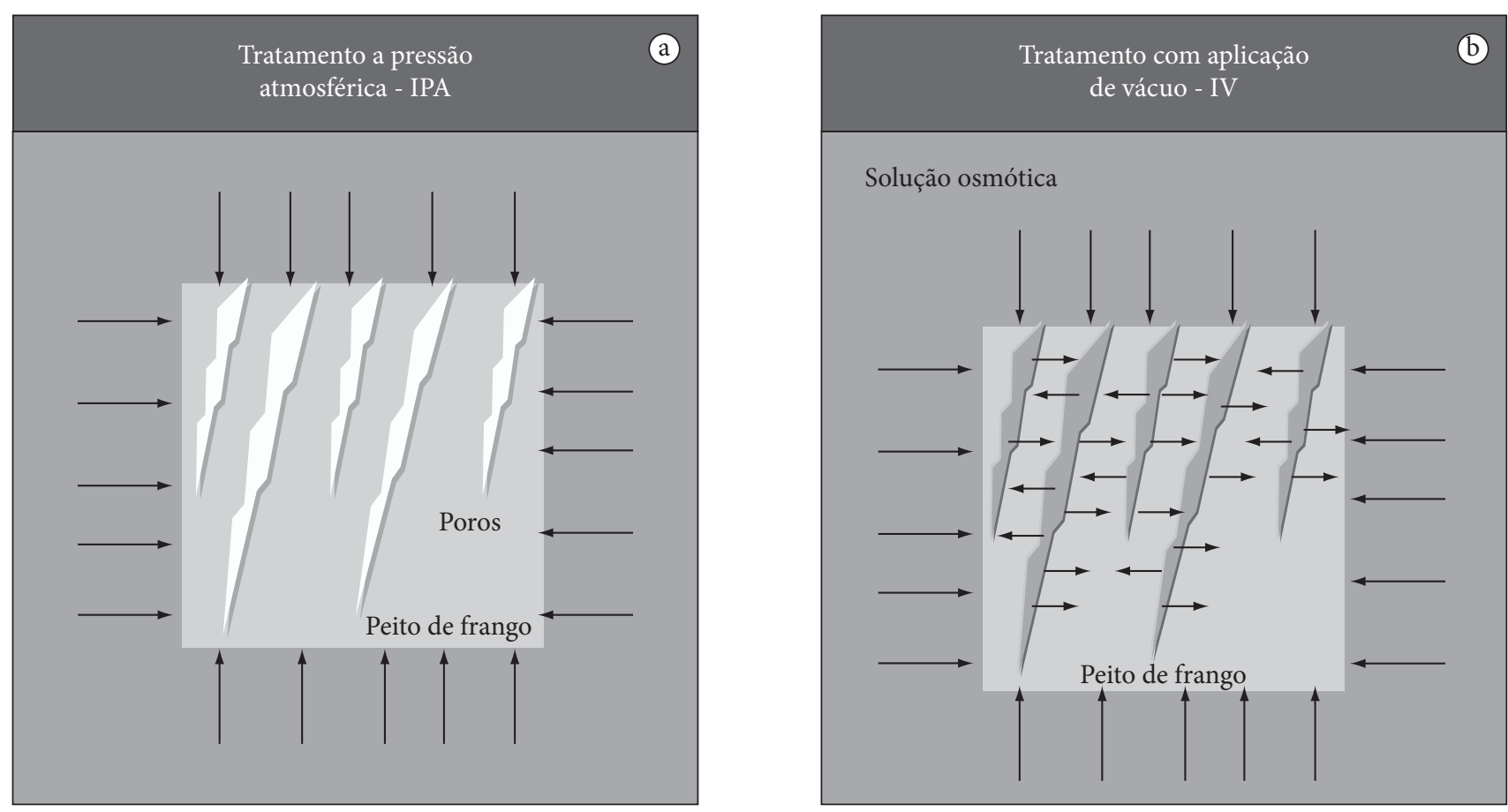

Figura 6. Ilustração da área disponível para transferência de massa no processo de salga de cortes de peito de frango por imersão, sendo: a) a pressão atmosférica (IPA); e b) com aplicação de vácuo (IV). 
Foi mostrado, através das Figuras $5 \mathrm{a}$, $5 \mathrm{~b}$ e $5 \mathrm{c}$, que a utilização de um período inicial de vácuo possibilitou o incremento da impregnação de solução salina durante o tratamento osmótico de peito de frango. Esse aumento foi devido à contribuição sinérgica do HDM com os mecanismos osmóticos e difusivos envolvidos no processo. O preenchimento do espaço intercelular pela solução externa provoca o aumento da área disponível para transferência de massa (FITO et al., 2001; SCHMIDT, 2006). O preenchimento dos espaços intercelulares acelera a formação do complexo sal-proteína, devido à maior área de contato. Por sua vez, a formação desse complexo contribui para aumento da porosidade do tecido muscular (BARAT et al., 2003; FOUCAT et al., 1995; OFFER; TRINICK, 1983) e, conseqüentemente, para uma maior entrada de solução por capilaridade.

\section{Conclusões}

A utilização de um período inicial de vácuo possibilita o incremento da impregnação de água e de sal no tratamento osmótico de peito de frango, devido à contribuição sinérgica do mecanismo hidrodinâmico (HDM) aos mecanismos osmóticos e difusivos envolvidos no processo.

Os dados experimentais de ganho de sal e de água podem ser representados matematicamente pelo modelo empírico utilizado.

A IV se apresenta como uma alternativa de processo para redução do tempo de salga de cortes de carne, mas deve-se tomar cuidado para que a incorporação de água e de sal esteja de acordo com os limites tecnológicos e legais.

\section{Referências bibliográficas}

ANDRÉS, A. M. G. Impregnacion a Vacio em Alimentos porosos. Aplicación al salado de quesos. Valencia, 1995, 195p. Tese (Doutorado em Tecnologia de Alimentos), Universidad Politécnica de Valencia.

AZUARA, E. et al. Kinetic model for osmotic dehydration and its relationship with Fick's second law. International Journal of Food Science and Technology, Reino Unido, v. 27, p. 409-418, 1992.

BARAT, J. M. et al. Post-salting studies in Spanish cured ham manufacturing. Time reduction by using brine thawing-salting. Meat Science, Estados Unidos, v. 69, n. 2, p. 201-208, 2005.

BARAT, J. M. et al. Cod salting manufacturing analysis. Food Research International, Canadá, v. 36, n. 5, p. 447-453, 2003.

BRASIL. Ministério da Agricultura e Abastecimento. Instrução Normativa no 20, de 21 de julho de 1999. Métodos Analíticos Físico-Químicos para Controle de Produtos Cárneos e seus
Ingredientes - Sal e Salmoura. Diário Oficial da União, Brasília, DF, 09 set. 1999.

Instrução Normativa $n^{\circ}$ 89, de 17 de dezembro de 2003. Regulamento Técnico de Qualidade e Identidade de Aves Temperadas. Diário Oficial da União, Brasília, DF, 18 dez. 2003.

CHIRALT, A. et al. Use of vacuum impregnation in food salting process. Journal of Food Engineering, Holanda, v. 49, n. 2-3, p. 141-151, 2001.

DEUMIER, F. et al. Pulsed vacuum brining of poultry meat: experimental study on the impact of vacuum cycles on mass transfer. Journal of Food Engineering, Holanda, v. 58, n. 1, p. $75-83,2003 a$.

Pulsed vacuum brining of poultry meat: interpretation of mass transfer mechanisms. Journal of Food Engineering, Holanda, v. 58 , n. 1, p. 85-93, 2003b.

FITO, P. Modelling of vacuum osmotic dehydration of food. Journal of Food Engineering, Holanda, v. 22, n. 1-4, p. 313-328, 1994.

FITO, P. et al. Coupling of hydrodynamic mechanism and deformationrelaxation phenomena during vacuum treatments in solid porous food-liquid systems. Journal of Food Engineering, Holanda, v. 27, n. 3, p. 229-240, 1996.

FITO, P. et al. Vacuum impregnation for development of new dehydrated products. Journal of Food Engineering, Holanda, v. 49, n. 4, p. 297-302, 2001.

FITO, P.; PASTOR, R. Non-diffusional mechanisms occurring during vacuum osmotic dehydration. Journal of Food Engineering, Holanda, v. 21, n. 4, p. 513-519, 1994.

FOUCAT, L. et al. Effect of brine injection on water dynamics in postmortem muscle: Study of T2 and diffusion coefficients by MR microscopy. Magnetic Resonance Imaging, Estados Unidos, v. 13, n. 2, p. 259-267, 1995.

HOFMEISTER, L. C; SOUZA, J. A. R.; LAURINDO, J. B. Use of dyed solutions to visualize different aspects of vacuum impregnation of Minas cheese. LWT-Food and Science Technology, Suiça, v. 38, n. 4, p. 379-386, 2005.

OFFER, G; TRINICK, J. On the mechanism of water holding in meat - the swelling and shrinking of myofibrils. Meat Science, Estados Unidos, v. 8, n. 4, p. 245-281, 1983.

PEZANTES, D. V. Estudo das operações combinadas da desidratação osmótica a vácuo, defumação líquida e secagem em filés de Bonito (Sarda sarda). Campinas, 2006. 211p. Tese (Doutorado em Engenharia de Alimentos), Faculdade de Engenharia de Alimentos, Universidade Estadual de Campinas (UNICAMP).

SCHMIDT, F. C. Estudo das trocas de massa durante o tratamento de cortes de peito de frango com soluções salinas. Florianópolis, 2006, 89p. Dissertação (Mestrado em Engenharia de Alimentos), Departamento de Engenharia Química e Engenharia de Alimentos, Universidade Federal de Santa Catarina (UFSC). 